\title{
Unified solver for fluid dynamics and aeroacoustics in isentropic gas flows
}

Article in Journal of Computational Physics · March 2017

DOI: 10.1016/j.jcp.2018.02.029

\section{CITATIONS}

0

4 authors:
READS

89
Arnau Pont Ribas
CIMNE International Center for Numerical Meth...

11 PubliCATIONS 10 CitATIONS

SEE PROFILE

Joan Baiges

Universitat Politècnica de Catalunya

41 PUBLICATIONS 292 CITATIONS

SEE PROFILE
Ramon Codina

Universitat Politècnica de Catalunya

224 PUBLICATIONS 5,741 CITATIONS

SEE PROFILE

Oriol Guasch

Universitat Ramon Llull

70 PUBLICATIONS 506 CITATIONS

SEE PROFILE

Some of the authors of this publication are also working on these related projects:

Project $\quad$ Extensive Unified-domain Simulation of the Human Voice View project

Project

Enhanced accuracy computational and experimental framework for strain localization and failure mechanisms (EACY) View project

All content following this page was uploaded by Joan Baiges on 05 September 2017. 


\title{
Unified solver for fluid dynamics and aeroacoustics in isentropic gas flows
}

\author{
Arnau Pont ${ }^{\mathrm{a}, *}$, Ramon Codina ${ }^{\mathrm{a}}$, Joan Baiges ${ }^{\mathrm{a}}$, Oriol Guasch ${ }^{\mathrm{b}}$ \\ ${ }^{a}$ International Centre for Numerical Methods in Engineering. C/ Gran Capita, s/n, \\ Campus Nord UPC, 08034 Barcelona, Catalonia, Spain \\ ${ }^{b}$ GTM Grup de recerca en Tecnologies Mèdia, La Salle, Universitat Ramon Llull. C/Quatre \\ Camins 30, 08022 Barcelona, Catalonia, Spain
}

\begin{abstract}
The propagation of acoustic waves is a physical phenomenon which can only be described taking into account the compressibility of the medium. The high computational cost of solving numerically the fully compressible Navier-Stokes equations, together with the poor performance of most numerical formulations for compressible flow in the low Mach number regime, has led to the necessity for more affordable numerical models for Computational Aeroacoustics. For low Mach number subsonic flows with neither shocks nor thermal coupling, both flow dynamics and wave propagation can be considered isentropic. Therefore, a joint isentropic formulation for flow and aeroacoustics can be devised which avoids the need for segregating flow and acoustic scales. Under these assumptions density and pressure fluctuations are directly proportional, and a two field velocity-pressure compressible formulation can be derived as an extension of an incompressible solver. On the other hand, the linear system of equations which arises from the proposed isentropic formulation is better conditioned than the homologous incompressible one due to the presence of a pressure perturbation term. Similarly to other compressible formulations the prescription of boundary conditions will have to deal with the backscattering of acoustic waves. In this sense, a separated imposition of boundary conditions for flow and acoustic scales which allows the evacuation of waves through Dirichlet boundaries
\end{abstract}

*Corresponding author: apont@cimne.upc.edu 
without using any tailored damping model will be presented.

Keywords: Isentropic flow, Computational Aeroacoustics, Numerical

Methods, Finite Elements, Lighthill Analogy

2017 MSC: 76Q05

\section{Introduction}

The compressibility behind the acoustics in Computational Fluid Dynamics (CFD) has been widely treated for several purposes along the history of numerical methods. Towards the 70's, the artificial compressibility method, [1,

5 was developed with the objective of reducing the computational cost of solving the incompressible Navier-Stokes equations in 3D domains, a research field which would also lead to projection methods, known nowadays as fractional step schemes. In this framework, the artificially added compressibility through a density or pressure perturbation term was not only a numerical artifact, but

10 a term that could be easily associated to the acoustics of a low speed compressible flow. However, the artificial compressibility method did not aim to describe the acoustic scales of the flow, but to introduce a numerical relaxation parameter which allowed an easier fulfillment of the continuity condition. The main modification of the incompressible Navier-Stokes consisted in adding an 15 artificial time derivative of the density or the pressure to the dimensionless continuity equation, which improved the condition number of the final system to be solved. A similar method was later applied by 2 to the low speed compressible Navier Stokes equations, in which a time derivative of the primitive variables was added to the energy equation in order to reduce the big disparity between the flow velocity and the sound speed. The Chorin method was extended for both incompressible and slow compressible flows by [3] by adding similar terms to all equations in order to obtain a symmetric hyperbolic problem. In other cases such as low Mach number $(M)$ compressible flows, the goal consisted precisely in going in the opposite direction and identifying the acoustic scales of the flow in order to remove them from the problem, 4, because they led to an 
ill-conditioning of the system and to the backscattering of sound waves into the computational domain.

While the addition of a certain amount of compressibility has made the calculation of incompressible flows easier without taking into account the consequent so acoustic field, the inclusion of compressibility in the flow formulation has been a drawback for calculating acoustics when dealing with low speed flows. The conservative compressible flow equations are considered the complete representation of the aeroacoustic problem because they describe directly all flow and acoustic scales without any need for modeling, which in terms of Computational 35 Fluid Dynamics (CFD) is called Direct Numerical Simulation (DNS), 5], and in acoustics is referred as Direct Noise Computation (DNC), 6]. However, as stated above, this formulation performs poorly for Mach numbers tending to zero due to the huge difference between flow velocity and wave propagation speed, which causes convergence problems. In order to avoid the bad conditioning of 40 the problem, a series of hybrid methods, which segregate the acoustics from the CFD, were developed. The so called acoustic analogies resolve the acoustic scales by means of an inhomogeneous wave equation where the source term that represents the aerodynamic noise comes from a previous flow calculation. The pioneer work in this field is presented in [7, which computes sound waves 45 propagation under the hypothesis of far-field flow conditions. The method has been progressively extended to include diffraction by solid boundaries [8] and moving surfaces [9]. Other hybrid methods, such as the incompressible-acoustic split method presented in [10, 11] enrich the incompressible flow equations with a variable density linked to pressure perturbations. Then, the time derivative of these perturbed density is translated into isentropic fluctuations of velocity and pressure that are propagated using a purely acoustic compressible solver after subtracting the incompressible component of the flow field. In a similar way, some formulations propagate the near field flow information to the far field with the Linearized Euler Equations (LEE), [12, 13, 14 or with the acoustic ${ }_{55}$ perturbation equations [15, 16, 17, which consist in an acoustic filtering of the LEE source term. All these methods allow a considerable flexibility, for example 
the use of a different discretization for each problem, as well as different flow and acoustic models. However, these models must be adapted to every case and the approximation errors need to be properly assessed. In some cases, acoustic source terms need to be modeled and might not be straightforward to implement in a FEM code. Moreover, the segregated calculation of the flow and acoustic components only assumes a one-way coupling from flow to acoustics, but not the other way around.

The formulation proposed in this work aims for a simplification of Computational Aeroacoustics (CAA) of isentropic compressible flows and proposes a general framework that can be applied to any geometry, spatial discretization or flow regime below the transonic range. It consists in a compressible formulation with primitive variables without solving for the energy equation, since the flow is considered to be isentropic, which after condensing the density field becomes a system of equations in terms of the velocity and the pressure, like in incompressible flow solvers. As a consequence, the implementation cost is very low when one departs from an already implemented incompressible flow solver. Also, the computational cost is reduced with respect to other methodologies due to the following reasons: getting rid of the fully compressible approach and 75 solving only for velocity and pressure, solving all scales at once without acoustic analogies and improving the condition number of the system the incompressible problem. The only drawback of such a compact system will be, of course, the lack of visualization of the acoustic fluctuations at the near field, where the aerodynamic scales are totally dominant and the wave propagation cannot be extracted like in [18] or [19]. As in all compressible flow models, an adequate equation of state needs to be chosen, in this case relating only density and pressure.

Since the present paper aims at solving both aerodynamics and acoustics scales in a single calculation, the prescription of compatible and accurate bound85 ary conditions for both components of the solution has been an important motivation for this work. From a numerical point of view, the imposition of boundary conditions can be performed as in the incompressible case, avoiding the difficul- 
ties found in compressible flows. However, omitting the acoustic scales in the treatment of the external boundaries leads to undesired wave reflections which affect the accuracy and the stability of the unified solver. Therefore, a new method including the combined imposition of essential boundary conditions in a weak sense on the mean flow variables, [20, and a Sommerfeld boundary condition for the acoustic component of the pressure will be presented, 21]. This combination will allow the acoustic wave to leave the domain through boundaries where the flow has been prescribed a certain boundary condition.

The paper is organized as follows: a detailed presentation of the isentropic compressible equations is shown in Section 2. The details of the aforementioned prescription of boundary conditions are presented in Section 3, and the stabilized time-discrete finite element formulation is derived in Section 4 . Finally, numerical results are presented in Section 5 two cases consisting in a 2D flow around a cylinder $(M=0.058)$ and a 3D flow around an airfoil $(M=0.4)$ will be presented and benchmarked against the Lighthill analogy, [7, with incompressible flow and the Ffowcs Williams Hawkings (FWH) acoustic analogy, 9, with a compressible formulation respectively. This analysis will allow to validate the present method in its whole application range.

\section{Problem formulation}

The present work focuses in the study of the aerodynamic and acoustic behavior of an ideal gas undergoing a reversible thermodynamical process, which is a realistic hypothesis in most aeroacoustic problems without heat transfer or shocks. This initial assumption allows a drastic simplification of the compressible Navier-Stokes equations, since the energy equation does not need to be solved and the primitive variables of the problem can be used. Moreover, a general formulation can be derived for both slow and high speed isentropic flows taking into account the following equalities, [22]:

$$
\frac{p_{0}}{p}=\left(1+\frac{\gamma-1}{2} M^{2}\right)^{\frac{\gamma}{\gamma-1}}
$$




$$
\frac{\rho_{0}}{\rho}=\left(1+\frac{\gamma-1}{2} M^{2}\right)^{\frac{1}{\gamma-1}}
$$

where $\gamma$ is the adiabatic constant of the gas, $p$ and $\rho$ are the total pressure and density fields including perturbations caused by the compressibility of the medium, whereas $p_{0}$ and $\rho_{0}$ are the same fields at stagnation conditions, [22]. $M$ is the Mach number, defined as:

$$
M:=\frac{|\mathbf{u}|}{c_{0}}
$$

where $|\mathbf{u}|$ is either the modulus of the pointwise velocity (or a characteristic value of it if one wants to define a global Mach number) and $c_{0}$ is the speed of sound in an ideal gas defined as $c_{0}=\sqrt{\frac{\gamma R T_{0}}{\mathcal{M}}}$, where $T_{0}$ is the temperature field at stagnation, $R[\mathrm{~J} / \mathrm{K} \cdot \mathrm{mol}]$ is the universal gas constant and $\mathcal{M}[\mathrm{kg} / \mathrm{mol}]$ is the molar mass of the gas. From Eq. (2), the following equality between both fields can be easily obtained:

$$
\frac{p_{0}}{p}=\left(\frac{\rho_{0}}{\rho}\right)^{\gamma}
$$

Then, deriving with respect to time both sides of Eq. (4) and using the equation of state for an ideal gas, $p_{0}=\frac{\rho_{0} R T_{0}}{\mathcal{M}}$, the next expression connecting pressure and density time derivatives can be found:

$$
\frac{\partial p}{\partial t}=\frac{p_{0}}{\rho_{0}} \gamma\left(1+\frac{\gamma-1}{2} M^{2}\right)^{-1} \frac{\partial \rho}{\partial t}=\frac{R T_{0}}{\mathcal{M}} \gamma\left(1+\frac{\gamma-1}{2} M^{2}\right)^{-1} \frac{\partial \rho}{\partial t}
$$

The final equation of state for a low speed gas flow can then be simplified to

$$
\frac{\partial p}{\partial t}=c_{0}^{2} \frac{\partial \rho}{\partial t}+\mathcal{O}\left(M^{2}\right)
$$

whereas for a compressible flow the speed of sound $c$ will have to be computed as follows:

$$
c^{2}=c_{0}^{2}\left(1+\frac{\gamma-1}{2} M^{2}\right)^{-1}
$$

and the following equation will hold:

$$
\frac{\partial p}{\partial t}=c^{2} \frac{\partial \rho}{\partial t}
$$


The same procedure can be applied to the pressure gradient obtaining the same relationship with respect to the density gradient. This explicit connection between pressure and density variations will allow to greatly simplify the compressible Navier-Stokes equations, since the density perturbations will be expressed in terms of the pressure. It is important to highlight that the limit $M \rightarrow 0$ will lead to a problem which will be very similar to the one resulting from the artificial compressibility method and will contain the acoustic scales of the flow. This is remarkable if it is compared to other non-isentropic formulations for low Mach numbers (see for instance [23]), where density variations are linked exclusively to temperature oscillations, and as a consequence no acoustics are captured.

Let us consider a computational domain $\Omega \subset \mathbb{R}^{d}$ (where $d=2,3$ is the number of space dimensions) with a domain boundary $\Gamma=\partial \Omega$ and let $(0, T)$ be the time interval of analysis. The isentropic compressible equations are then:

$$
\begin{array}{r}
\rho \frac{\partial \mathbf{u}}{\partial t}+\rho(\mathbf{u} \cdot \nabla) \mathbf{u}-\mu \nabla^{2} \mathbf{u}-\frac{1}{3} \mu \nabla(\nabla \cdot \mathbf{u})+\nabla p=\mathbf{0} \text { in } \Omega, \\
\partial_{t} \rho+\mathbf{u} \cdot \nabla \rho+\rho \nabla \cdot \mathbf{u}=0 \text { in } \Omega,
\end{array}
$$

where $\mathbf{u}$ is the velocity, $p$ the pressure and $\mu$ the dynamic viscosity. Boundary and initial conditions need to be appended to this problem. Using Eq. (5) $\rho$ can be expressed in terms of $p$ and the continuity equation becomes

$$
\frac{1}{c^{2}} \partial_{t} p+\frac{1}{c^{2}} \mathbf{u} \cdot \nabla p+\rho \nabla \cdot \mathbf{u}=0 \text { in } \Omega
$$

where $c(\mathbf{x}, t)$ is given by Eq. (7) and $\mathbf{x}$ is the spatial coordinate vector. Despite all simplifications, the previous equation still depends on the function $c$ and two density dependent terms remain in the momentum equation. Calculating these two fields as implicit functions of $(\mathbf{u}, p)$ would increase the complexity of the new scheme with new non-linearities. In this sense, the finite element approximation will include the necessary elimination of these variables in order to obtain a problem depending only on the velocity and pressure fields.

The next step consists in deriving the variational formulation of the previous problem. Let us denote with $\langle\cdot, \cdot\rangle_{\omega}$ the integral of the product of two functions 
in the domain $\omega$ (with the subscript omitted when $\omega=\Omega$ ) and $(\cdot, \cdot)$ the $L^{2}(\Omega)$ inner product. Let $V$ and $Q$ be the functional spaces where for each time $t$ the velocity and pressure solutions live respectively with appropriate regularity that we will not analyze here. Then, defining the velocity and pressure test functions $\mathbf{v} \in V$ and $\rho q \in Q$ the variational formulation can be written in terms of the forms:

$$
\begin{aligned}
B([\mathbf{u}, p],[\mathbf{v}, q])= & \left(\rho \mathbf{v}, \partial_{t} \mathbf{u}\right)+\langle\rho \mathbf{v},(\mathbf{u} \cdot \nabla) \mathbf{u}\rangle+\mu(\nabla \mathbf{v}, \nabla \mathbf{u}) \\
& +\frac{1}{3} \mu(\nabla \cdot \mathbf{v}, \nabla \cdot \mathbf{u})-(\nabla \cdot \mathbf{v}, p) \\
& +\left(\frac{1}{c^{2}} q, \partial_{t} p\right)+\left(\frac{1}{c^{2}} q, \mathbf{u} \cdot \nabla p\right)+(\rho q, \nabla \cdot \mathbf{u}), \\
\tilde{B}_{B}([\mathbf{u}, p],[\mathbf{v}, q])= & -\langle\mathbf{v}, \mathbf{n} \cdot \sigma(\mathbf{u}, p)\rangle_{\Gamma},
\end{aligned}
$$

where $B$ and $\tilde{B}$ are two forms and the stress tensor is defined as $\sigma(\mathbf{u}, p)=$ $-p \mathbf{I}+\mu \nabla \mathbf{u}+\frac{1}{3} \mu(\nabla \cdot \mathbf{u}) \mathbf{I}$.

The Galerkin weak form of the problem prior to applying boundary conditions can be written as follows: for all time $t>0$, find $\mathbf{u} \in V$ and $p \in Q$, with appropriate regularity in time, such that:

$$
B([\mathbf{u}, p],[\mathbf{v}, q])+\tilde{B}_{B}([\mathbf{u}, p],[\mathbf{v}, q])=0
$$

for all $\mathbf{v} \in V$ and $\rho q \in Q$. Equations (2) and (7) are used to close the problem. Moreover, initial conditions need to be appended. Boundary conditions will be defined in the following section proposing a new formulation for the form $\tilde{B}_{B}$. This will give rise to a decomposition of the form $\tilde{B}_{B}=B_{B}-L_{B}$, with $B_{B}$ depending on the unknowns and $L_{B}$ on the boundary data, so that it can be moved to the right-hand-side of 15 .

\section{Imposition of boundary conditions}

\subsection{Mean and acoustic components}

Although the intricate prescription of boundary conditions of the fully compressible formulation is avoided in the present problem, new challenges arise 
which need to be accounted for. Given that flow and acoustic scales need to be resolved together, and that the domain must be truncated at some point in order to bound the computational cost, an appropriate boundary condition for the acoustic component of the pressure must be used. The main objective of this boundary conditions is to avoid the sound waves being backscattered by the external boundaries into the computational domain. There are several numerical methods which deal with this problem, see for instance the reviews in [24] and [25]. Here we highlight some of the most relevant ones: in [26] a nonreflecting boundary condition (NRBC) was presented for the Euler equations in multi-dimensional domains which modeled the waves using the characteristic equations. However, the identification of waves is not so straightforward in the Navier-Stokes equations and the assumption of one-dimensional flow on the boundaries had to be made [27]. This method yields proper results in boundaries where the solution is homogeneous and known, such as the inlet. In [28], 29 and 30 the method was extended using low Mach number asymptotics in order to account for viscous and transverse effects on the wave. Another family of methods are the so called Perfectly Matched Layer (PML) methods, see [31. and 32 , which can work under near-field conditions and thus allow for smaller computational domains to be used. These methods use an auxiliary domain beyond the outer boundaries which absorbs the incident waves without reflecting them back. A third alternative for the non-reflection of waves are the radiative and outflow conditions developed in 33 and extended in 34. Similarly to the PML method, they also consider a secondary domain, but in this case a modified set of equations minimizing reflection is solved at the far-field.

On the other hand, the treatment of the waves must be compatible with the flow velocity boundary conditions. This is of most importance on Dirichlet boundaries where the velocity needs to be prescribed. This need has motivated the development of a novel method for a unified prescription of flow and nonreflecting boundary conditions, which will be presented next. The method is able to deal with subsonic flows solved in arbitrary geometries, and following the aim of being a general formulation, it is compatible with any non-reflecting 
model for the acoustic waves, which will not be part of the work scope. The development of the method starts by splitting the two fields of the problem into mean variables $\overline{\mathbf{u}}$ and $\bar{p}$, and oscillatory components $\mathbf{u}^{\prime}$ and $p^{\prime}$ as follows:

$$
\begin{aligned}
& \mathbf{u}(\mathbf{x}, t)=\overline{\mathbf{u}}(\mathbf{x}, t)+\mathbf{u}^{\prime}(\mathbf{x}, t), \\
& p(\mathbf{x}, t)=\bar{p}(\mathbf{x}, t)+p^{\prime}(\mathbf{x}, t),
\end{aligned}
$$

where

$$
\begin{aligned}
\overline{\mathbf{u}}(\mathbf{x}, t) & :=\frac{1}{T_{w}} \int_{t-T_{w}}^{t} \mathbf{u}(\mathbf{x}, s) d s \\
\bar{p}(\mathbf{x}, t) & :=\frac{1}{T_{w}} \int_{t-T_{w}}^{t} p(\mathbf{x}, s) d s
\end{aligned}
$$

and $T_{w}$ is an appropriate time window. The mean flow variables are allowed to evolve during the calculation and they do not necessary need to be homogeneous along the boundary, but high frequency variations of these variables are not allowed because they would interfere with the acoustic fluctuations $\left(\mathbf{u}^{\prime}, p^{\prime}\right)$.

\subsection{Split boundary conditions}

In order to treat flow and acoustic boundary conditions in an appropriate way, the boundary $\Gamma$ has been divided into three disjoint subsets $\Gamma_{S}, \Gamma_{L}$ and $\Gamma_{O}$, which will refer to the solid reflecting boundaries where velocity is prescribed to zero, the lateral walls and the outflow, respectively. Whereas the first and the latter ones have a clear physical meaning, $\Gamma_{L}$ is defined for numerical convenience: it is composed of any outer boundary with at least one component of the velocity prescribed to a known value, which means that it also encompasses the inlet boundaries, see Fig. 1. The upper and lower walls belong to $\Gamma_{L}$ because they have been assumed to be a mere truncation of the computational domain. This artificial truncation of the domain, which is performed with the objective of limiting the computational cost, does not assume that the affected boundaries are part of the outflow because this may not properly represent the physics of the flow and could even lead to numerical instabilities. Therefore, the flow needs to be confined without affecting the outward propagation of the 
waves. This boundary definition has been used for the two cases presented in the Section 5

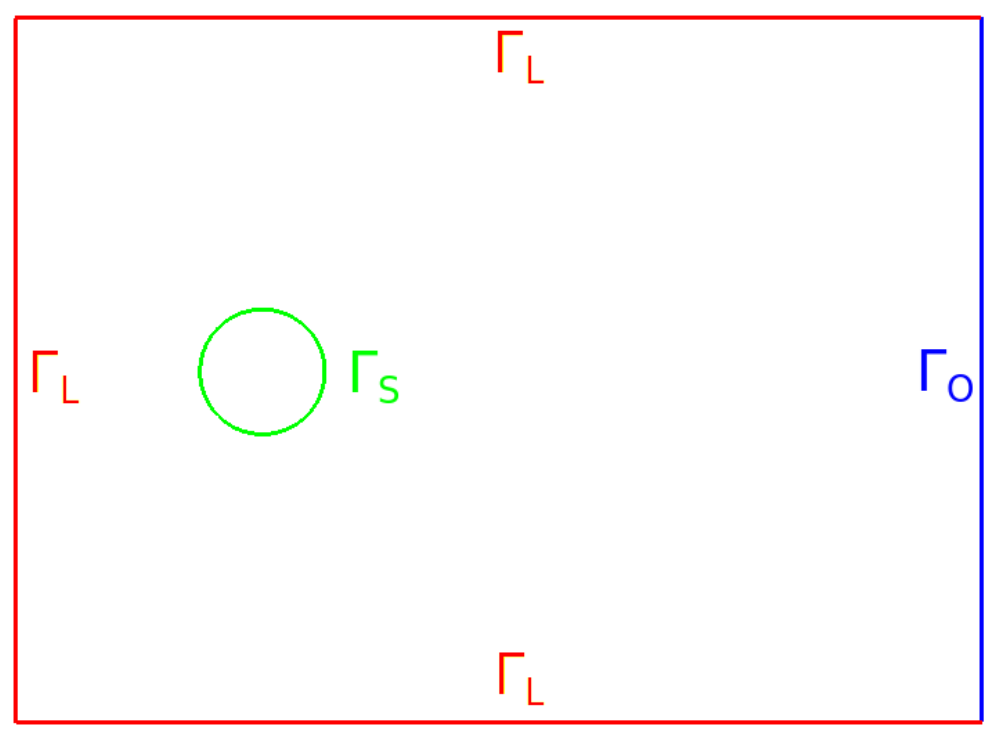

Figure 1: Schematic definition of domain boundaries.

On $\Gamma_{L}$ and $\Gamma_{O}$, the far-field conditions assume that the acoustic scales are dominant. This means that a naive approach using a zero traction boundary condition would lead to backscatter and reflection of the exiting waves into the domain. Therefore, a non-reflecting numerical model must be applied on these boundaries, see for example 21].

The proposed methodology for applying compatible flow and acoustic boundary conditions on $\Gamma_{L}$ and $\Gamma_{O}$ is based on a weak or weighted prescription of the Dirichlet conditions together with the use of a Sommerfeld type boundary condition. The boundary conditions for the problem can be formulated as follows:

On the solid boundary $\Gamma_{S}$, where the velocity is known and the incident waves are expected to reflect, we enforce:

$$
\mathbf{u}=\overline{\mathbf{u}}+\mathbf{u}^{\prime}=\mathbf{u}_{S} \text { on } \Gamma_{S},
$$

where $\mathbf{u}_{S}$ is the prescribed velocity on the solid boundary. 
On the truncation boundary $\Gamma_{L}$, several conditions are going to be enforced: firstly, the mean value of the velocity is going to be prescribed to the inlet (or truncation) boundary velocity $\mathbf{u}_{L}$ :

$$
\overline{\mathbf{u}}=\mathbf{u}_{L} \quad \text { on } \Gamma_{L},
$$

Secondly, a Sommerfeld-like boundary condition for the fluctuating part of the velocity and pressure fields is prescribed:

$$
\mathbf{n} \cdot \mathbf{u}^{\prime}=-\frac{1}{c \rho} \mathbf{n} \cdot\left[\mathbf{n} \cdot \sigma\left(\mathbf{u}^{\prime}, p^{\prime}\right)\right] \quad \text { on } \Gamma_{L}
$$

where $\mathbf{n}$ is the unit outward normal to $\Gamma_{L}$. Let also be $\mathbf{m}$ any unit vector tangent to it. The fluctuating tractions in the tangential direction are prescribed to zero:

$$
\mathbf{m} \cdot\left[\mathbf{n} \cdot \sigma\left(\mathbf{u}^{\prime}, p^{\prime}\right)\right]=0 \text { on } \Gamma_{L} .
$$

Finally, on the outflow boundary $\Gamma_{O}$, the following conditions are going to be applied:

$$
\mathbf{n} \cdot \sigma(\overline{\mathbf{u}}, \bar{p})=\mathbf{t}_{O} \quad \text { on } \Gamma_{O}
$$

which enforces the mean value tractions to the prescribed value $t_{O}$. Regarding the fluctuating values, the same approach used for $\Gamma_{L}$ is used, with a Sommerfeld-like condition in the normal direction and zero traction prescribed in the tangential directions:

$$
\begin{aligned}
\mathbf{n} \cdot \mathbf{u}^{\prime} & =-\frac{1}{c \rho} \mathbf{n} \cdot\left[\mathbf{n} \cdot \sigma\left(\mathbf{u}^{\prime}, p^{\prime}\right)\right] \quad \text { on } \Gamma_{O}, \\
\mathbf{m} \cdot\left[\mathbf{n} \cdot \sigma\left(\mathbf{u}^{\prime}, p^{\prime}\right)\right] & =0 \text { on } \Gamma_{O} .
\end{aligned}
$$

Note that:

- $\Gamma_{S}$ is a classical Dirichlet-type boundary. Velocity test functions will vanish there and the condition $\mathbf{u}=\mathbf{u}_{S}$ can be prescribed in a strong way.

- $\Gamma_{L}$ is a boundary where Dirichlet-type boundary conditions are prescribed for $\overline{\mathbf{u}}$ and mixed boundary conditions for $\mathbf{u}^{\prime}$, namely the normal component and the tangent associated stress. Both will be prescribed weakly. 
- $\Gamma_{O}$ is a boundary where Neumann-type boundary conditions are prescribed for $\overline{\mathbf{u}}$ and mixed boundary conditions for $\mathbf{u}^{\prime}$, the same as on $\Gamma_{L}$. All these conditions will be prescribed weakly.

- For inviscid flows, the condition on the normal component of $\mathbf{u}^{\prime}$ reduces to Sommerfeld's condition $p^{\prime}=c \rho\left(\mathbf{u}^{\prime} \cdot \mathbf{n}\right)$. Obviously, other non-reflecting boundary conditions would be used.

Let us see how to prescribe these boundary conditions in the variational form of the problem. Let us start by noting that

$$
\begin{aligned}
-\langle\mathbf{v}, \mathbf{n} \cdot \sigma(\mathbf{u}, p)\rangle_{\Gamma}= & -\langle\mathbf{v}, \mathbf{n} \cdot \sigma(\mathbf{u}, p)\rangle_{\Gamma_{L}}-\langle\mathbf{v}, \mathbf{n} \cdot \sigma(\mathbf{u}, p)\rangle_{\Gamma_{O}} \\
= & -\langle\mathbf{v}, \mathbf{n} \cdot \sigma(\overline{\mathbf{u}}, \bar{p})\rangle_{\Gamma_{L}}-\left\langle\mathbf{v}, \mathbf{n} \cdot \sigma\left(\mathbf{u}^{\prime}, p^{\prime}\right)\right\rangle_{\Gamma_{L}} \\
& -\langle\mathbf{v}, \mathbf{n} \cdot \sigma(\overline{\mathbf{u}}, \bar{p})\rangle_{\Gamma_{O}}-\left\langle\mathbf{v}, \mathbf{n} \cdot \sigma\left(\mathbf{u}^{\prime}, p^{\prime}\right)\right\rangle_{\Gamma_{O}} \\
= & -\langle\mathbf{v}, \mathbf{n} \cdot \sigma(\overline{\mathbf{u}}, \bar{p})\rangle_{\Gamma_{L}}+\left\langle c \rho \mathbf{v} \cdot \mathbf{n}, \mathbf{u}^{\prime} \cdot \mathbf{n}\right\rangle_{\Gamma_{L}} \\
& -\left\langle\mathbf{v}, \mathbf{t}_{O}\right\rangle_{\Gamma_{O}}+\left\langle c \rho \mathbf{v} \cdot \mathbf{n}, \mathbf{u}^{\prime} \cdot \mathbf{n}\right\rangle_{\Gamma_{O}}
\end{aligned}
$$

Note that no contribution on $\Gamma_{S}$ has been included, since in this boundary usual Dirichlet boundary conditions are applied and the test functions vanish on it. We still need to prescribe $\overline{\mathbf{u}}=\mathbf{u}_{L}$, which has to be done through penalization using Nitsche's method, 35. In the spirit of this method, it is convenient to symmetrize the boundary terms. Taking this into account we define the boundary terms, which can be written as $B_{B}([\mathbf{u}, p],[\mathbf{v}, q])-L_{B}([\mathbf{v}, q])$, with

$$
\begin{aligned}
B_{B}([\mathbf{u}, p],[\mathbf{v}, q]):= & -\langle\mathbf{v}, \mathbf{n} \cdot \sigma(\overline{\mathbf{u}}, \bar{p})\rangle_{\Gamma_{L}}-\langle\overline{\mathbf{u}}, \mathbf{n} \cdot \sigma(\mathbf{v}, q)\rangle_{\Gamma_{L}}+\beta \frac{\mu_{p}}{l_{p}}\langle\mathbf{v}, \overline{\mathbf{u}}\rangle_{\Gamma_{L}} \\
& +\left\langle c \rho \mathbf{v} \cdot \mathbf{n}, \mathbf{u}^{\prime} \cdot \mathbf{n}\right\rangle_{\Gamma_{L}}+\left\langle c \rho \mathbf{v} \cdot \mathbf{n}, \mathbf{u}^{\prime} \cdot \mathbf{n}\right\rangle_{\Gamma_{O}} \\
L_{B}([\mathbf{v}, q]):= & -\left\langle\mathbf{u}_{L}, \mathbf{n} \cdot \sigma(\mathbf{v}, q)\right\rangle_{\Gamma_{L}}+\beta \frac{\mu_{p}}{l_{p}}\left\langle\mathbf{v}, \mathbf{u}_{L}\right\rangle_{\Gamma_{L}}+\left\langle\mathbf{v}, \mathbf{t}_{O}\right\rangle_{\Gamma_{O}}
\end{aligned}
$$

where $\beta, \mu_{p}, l_{p}$ are numerical parameters, the first one dimensionless, the second one with units of viscosity and the latter with units of length. We are still at the continuous level. When a finite element approximation in space is performed, $h$ being the element size, one can show that $\mu_{p}, l_{p}$ can be taken as $\mu_{p}=\mu+|\mathbf{u}| h$, and $l_{p}=h,[36]$. 
The only ingredient missing in the formulation is to define the outflow traction $\mathbf{t}_{O}$. Assuming that $\Gamma_{O}$ is placed in a far-field region, where $\bar{p} \approx 0$ and $\nabla \overline{\mathbf{u}} \approx 0$, then the natural condition to be imposed is $\mathbf{t}_{O}=\mathbf{0}$.

\subsection{Domain truncation}

The truncation of the domain is a problematic issue when dealing with acoustic waves. Sometimes, especially in case of low speed flows, the far field conditions are reached within a small distance of the solid objects causing the perturbation. In this cases the truncation of the domain will only depend on the measure of the largest wavelength. Therefore, in such scenarios the present formulation can be applied in a general way without further artifacts. However, when convection becomes dominant stagnation conditions may be found far away from the perturbation, which results in a high computational cost if the full near-field domain needs to be simulated. Moreover, many times the Sommerfeld non-radiating boundary condition is compromised, since it assumes an orthogonal incidence of the wave front with the external boundary.

An example illustrating this situation is depicted in Fig. 2, In this case a $M=0.4$ flow over a wing profile is calculated departing from a fully developed flow solution. Before any wave reaches the boundary, the outlet is already reflecting the noise produced by the vortices passing through it, see Fig. 2 .

In the second numerical example shown in Section 5 , this problem has been solved by adding a spherical PML based on [37] in an artificial outlet domain $\Omega_{P M L}$, see Fig. 3. For this, we define the finite element contribution of the PML layer in a new bilinear form $B_{P M L}$ :

$$
B_{P M L}([\mathbf{u}, p],[\mathbf{v}, q]):=\left(\mathbf{v}, \alpha^{*} \mathbf{u}\right)+(q, \rho \alpha p)
$$

where $\alpha^{*}$ is defined as:

$$
\begin{aligned}
\alpha(r)=0.4 \frac{\left(r-r_{0}\right)^{2}}{\left(r_{f}-r_{0}\right)^{3}}\left(-2 r+3 r_{f}-r_{0}\right) & \text { in } \Omega_{P M L}, \\
\alpha(r)=0 & \text { in } \Omega \backslash \Omega_{P M L},
\end{aligned}
$$




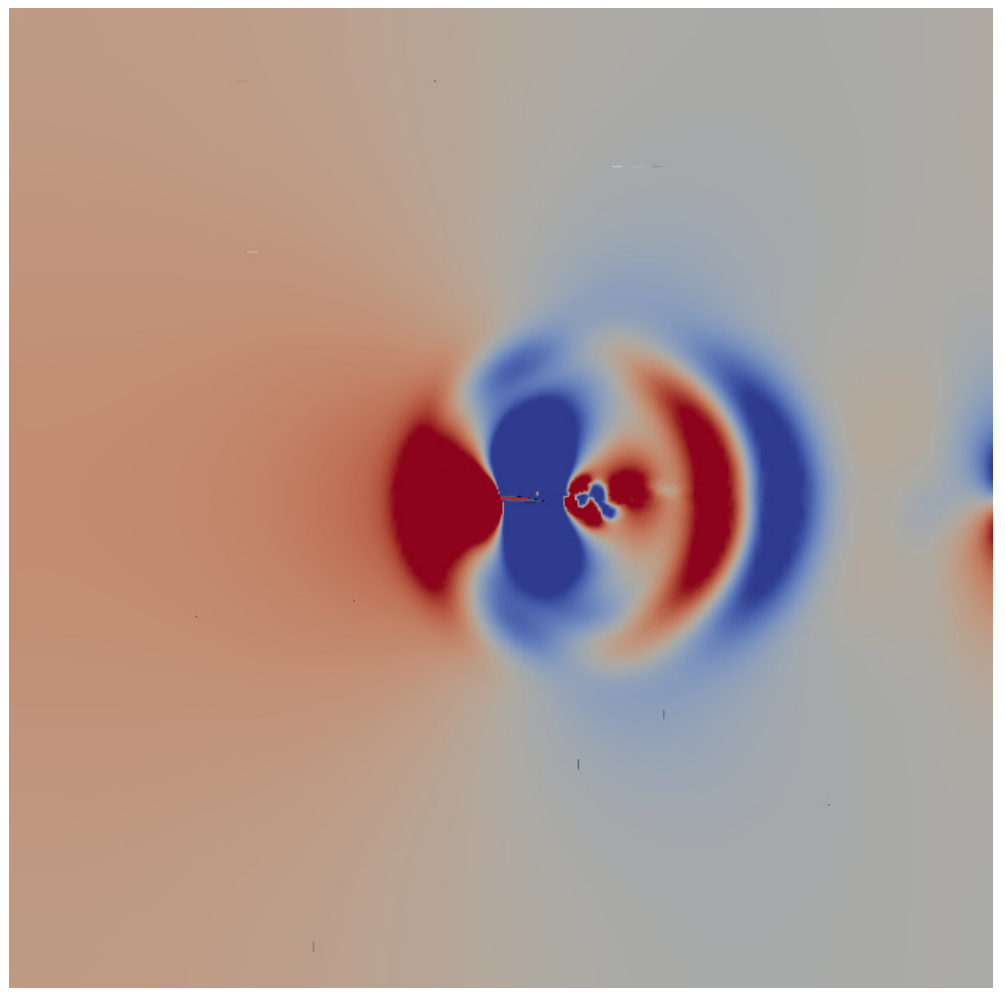

Figure 2: Reflection of the sound generated by vortices approaching the outflow.

where $\alpha^{*}=\alpha \rho^{2} c^{2}$ and $r_{0}$ and $r_{f}$ are the small and big radius of the PML, respectively. Finally, the problem to be solved will be in this case

$$
B([\mathbf{u}, p],[\mathbf{v}, q])+B_{P M L}([\mathbf{u}, p],[\mathbf{v}, q])+B_{B}([\mathbf{u}, p],[\mathbf{v}, q])=L_{B}([\mathbf{v}, q])
$$

Unlike Section 3 the importance of absorbing both hydrodynamic and acoustic scales on the outlet justifies the application of the PML to the whole variables $\left(\mathbf{u}_{h}^{n+1}, p_{h}^{n+1}\right)$. The performance of this numerical tool will be presented in the Section 5 ,

\section{Numerical approximation}

In this section we present the finite element formulation for the space approximation of the isentropic Navier-Stokes equations, including the stabilization 


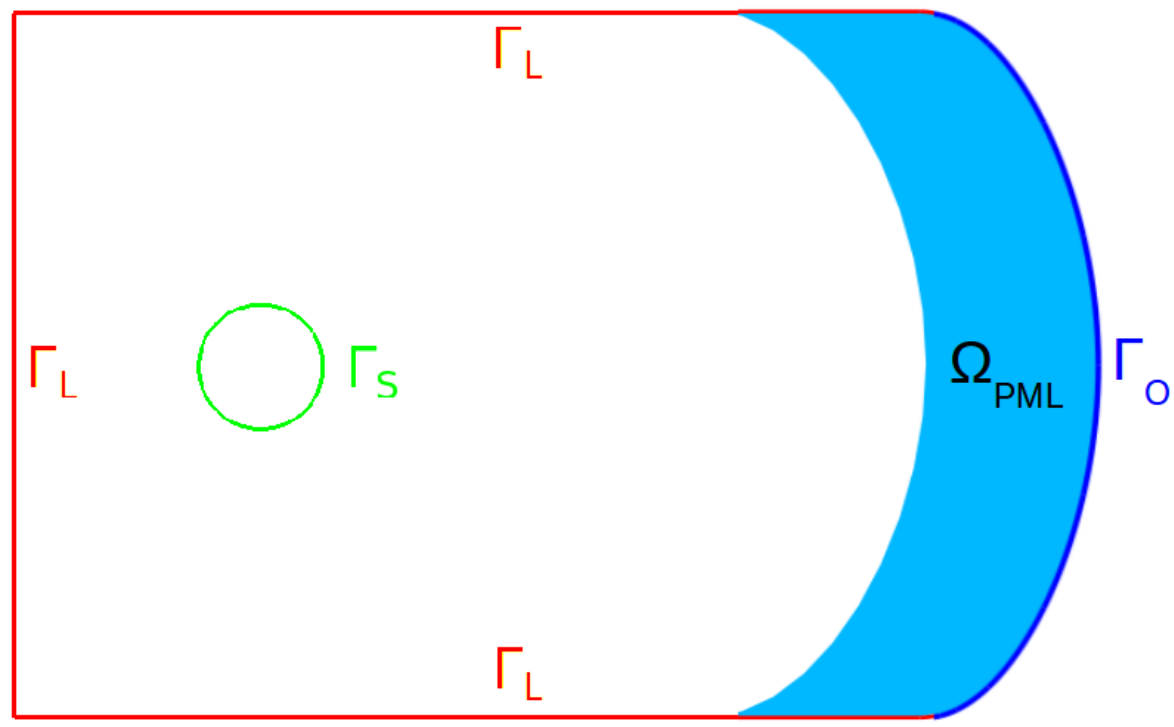

Figure 3: A PML is attached to the original outlet of the domain $\Omega$.

terms required for obtaining a stable formulation when using P1/P1 velocitypressure elements, as well as the time discretization using finite differences.

Let us consider a finite element partition of the domain $\Omega$ of size $h$, and use this letter as subscript to denote finite element functions and spaces. Only conforming finite element approximations will be considered in what follows. Let $V_{h} \subset V$ be the finite approximation space for the discrete velocity field and let us also define $Q_{h} \subset Q$, the pressure approximation space.

\subsection{Time discretization}

Concerning the time integration, the monolithic approach for solving the incompressible Navier-Stokes equations consists in building a system with both velocity and pressure degrees of freedom, which leads to the coupled calculation of the momentum and mass equations in one single step. To approximate the first order time derivatives, a second order backward finite difference scheme 
(BDF2) has been used. Let us partition the time interval $[0, T]$ into $N$ equal time steps of size $\delta t:=t^{n+1}-t^{n}$ so that $0 \equiv t^{0}<t^{1}<\ldots<t^{n}<\ldots<t^{N} \equiv T$. Given a generic time dependent function $g(t)$, the following notation will be used for the BDF2 approximation to the first time derivative:

$$
\left.\partial_{t} g\right|_{t^{n+1}} \approx \delta_{t} g^{n+1}:=\frac{1}{\delta t}\left(\frac{3}{2} g^{n+1}-2 g^{n}+\frac{1}{2} g^{n-1}\right),
$$

where $g^{n}$ denotes evaluation of $g$ at time step $t^{n}$.

\subsection{Discrete boundary conditions}

At an arbitrary time step of the numerical simulation, the final fully discretized implicit scheme in space and time can be derived using the finite element formulation described below. Moreover, the mean flow values must be expressed according to the chosen integration scheme and the penalty parameters of the weak essential condition on $\Gamma_{L}$ must be defined. We do this as follows:

- As mentioned above, $\mu_{p}, l_{p}$ can be taken as $\mu_{p}=\mu+|\mathbf{u}| h, l_{p}=h, 36$.

- If the temporal window presented at Eq. 18 is defined at a discrete level as $T_{w}=N_{w} \delta t$ and we use the trapezoidal rule for the integration, then the mean values can be expressed as follows:

$$
\overline{\mathbf{u}}_{h}^{n+1}=\frac{\delta t}{T_{w}}\left(\frac{1}{2} \mathbf{u}_{h}^{n+1}+\sum_{k=n-N_{w}+2}^{n} \mathbf{u}_{h}^{k}+\frac{1}{2} \mathbf{u}_{h}^{n-N_{w}+1}\right)
$$

Bearing in mind the sharp initial pressure transient and the absence of a minimally developed mean flow, it is important to run several time steps $\left(N_{w}\right)$ before using the present formulation in order to obtain representative mean flow variables. The same procedure is applied to $p$ and the fluctuating components will be also expressed now on in terms of the full variables evaluated at $t^{n+1}$.

\subsection{Finite element approximation}

For a better understanding of the derivation, the formulation will be arranged in five forms: $\mathcal{B}, \mathcal{B}_{B}, \mathcal{B}_{P M L}, \mathcal{L}_{B}$ and $\mathcal{B}_{S}$, which corresponds to the Algebraic Subgrid Scale (ASGS) stabilization terms and will be presented next. The 
final formulation reads as follows: from known $\mathbf{u}_{h}^{n-2}, \mathbf{u}_{h}^{n-1}$ and $\mathbf{u}_{h}^{n}$, compute the compressible velocity and pressure at time step $t^{n+1},\left[\mathbf{u}_{h}^{n+1}, p_{h}^{n+1}\right] \in \mathcal{V}_{h} \times \mathcal{Q}_{h}$, such that

$$
\begin{array}{r}
B\left(\left[\mathbf{u}_{h}, p_{h}\right],\left[\mathbf{v}_{h}, q_{h}\right]\right)+B_{P M L}\left(\left[\mathbf{u}_{h}, p_{h}\right],\left[\mathbf{v}_{h}, q_{h}\right]\right)+B_{B}\left(\left[\mathbf{u}_{h}, p_{h}\right],\left[\mathbf{v}_{h}, q_{h}\right]\right) \\
+B_{S}\left(\left[\mathbf{u}_{h}, p_{h}\right],\left[\mathbf{v}_{h}, q_{h}\right]\right)=L_{B}\left(\left[\mathbf{v}_{h}, q_{h}\right]\right),
\end{array}
$$

for all test functions, where

$$
\begin{array}{r}
B\left(\left[\mathbf{u}_{h}, p_{h}\right],\left[\mathbf{v}_{h}, q_{h}\right]\right)=\left(\rho^{n+1} \mathbf{v}_{h}, \delta_{t} \mathbf{u}_{h}^{n+1}\right)+\left\langle\rho^{n+1} \mathbf{v}_{h},\left(\mathbf{u}_{h}^{n+1} \cdot \nabla\right) \mathbf{u}_{h}^{n+1}\right\rangle \\
+\mu\left(\nabla \mathbf{v}_{h}, \nabla \mathbf{u}_{h}^{n+1}\right)+\frac{1}{3} \mu\left(\nabla \cdot \mathbf{v}_{h}, \nabla \cdot \mathbf{u}_{h}^{n+1}\right)-\left(\nabla \cdot \mathbf{v}_{h}, p_{h}^{n+1}\right) \\
+\left(\frac{1}{\left(c^{2}\right)^{n+1}} q_{h}, \delta_{t} p_{h}^{n+1}\right)+\left(\frac{1}{\left(c^{2}\right)^{n+1}} q_{h}, \mathbf{u}_{h}^{n+1} \cdot \nabla p_{h}^{n+1}\right)+\left(\rho^{n+1} q_{h}, \nabla \cdot \mathbf{u}_{h}^{n+1}\right) .
\end{array}
$$

As mentioned before, the condensation of $\rho^{n+1}$ and $c^{n+1}$ is essential for keeping the complexity of the formulation low. For this reason, these two variables have been included in non-linearity iterative loop so they are updated at every time step at each Gauss point. In this way, they are evaluated with the converged unknowns of the problem at $t^{n+1}$, as the implicit scheme requires:

$$
\begin{gathered}
\rho^{n+1}=\rho_{0}\left(1+\frac{\gamma-1}{2} \frac{\left|\mathbf{u}_{h}^{n+1}\right|^{2}}{c_{0}^{2}}\right)^{\gamma-1}, \\
\left(c^{2}\right)^{n+1}=c_{0}^{2}\left(1+\frac{\gamma-1}{2} \frac{\left|\mathbf{u}_{h}^{n+1}\right|^{2}}{c_{0}^{2}}\right)^{-1} .
\end{gathered}
$$

Since it is understood that $\rho$ and $c$ are only evaluated at $t^{n+1}$, now on they will be referred as $\rho$ and $c$ instead of $\rho^{n+1}$ and $\left(c^{2}\right)^{n+1}$.Next, the bilinear form $B_{B}$ and the linear form $L_{B}$ can be easily obtained using (21):

$$
\begin{aligned}
B_{B}\left(\left[\mathbf{u}_{h}, p_{h}\right],\left[\mathbf{v}_{h}, q_{h}\right]\right)= & -\left\langle\mathbf{v}_{h}, \mathbf{n} \cdot \sigma_{h}\left(\overline{\mathbf{u}}_{h}^{n+1}, \bar{p}_{h}^{n+1}\right)\right\rangle_{\Gamma_{L}}-\left\langle\overline{\mathbf{u}}_{h}^{n+1}, \mathbf{n} \cdot \sigma_{h}\left(\mathbf{v}_{h}, q_{h}\right)\right\rangle_{\Gamma_{L}} \\
& +\beta \frac{\mu_{p}}{l_{p}}\left\langle\mathbf{v}_{h}, \overline{\mathbf{u}}_{h}^{n+1}\right\rangle_{\Gamma_{L}}+\left\langle c \rho \mathbf{v}_{h} \cdot \mathbf{n}, \mathbf{u}_{h}^{\prime n+1} \cdot \mathbf{n}\right\rangle_{\Gamma_{L} \cup \Gamma_{O}}, \\
L_{B}\left(\left[\mathbf{v}_{h}, q_{h}\right]\right)= & \beta \frac{\mu_{p}}{l_{p}}\left\langle\mathbf{v}_{h}, \mathbf{u}_{L}\right\rangle_{\Gamma_{L}}-\left\langle\mathbf{u}_{L}, \mathbf{n} \cdot \sigma_{h}\left(\mathbf{v}_{h}, q_{h}\right)\right\rangle_{\Gamma_{L}},
\end{aligned}
$$

where we have assumed that $\mathbf{t}_{O}=\mathbf{0}$. Applying the definition of the mean values presented in Eq. (26) and expressing the fluctuating components in terms of the 
problem unknowns, $B_{B}$ can be rewritten as follows:

$$
\begin{array}{r}
B_{B}\left(\left[\mathbf{u}_{h}, p_{h}\right],\left[\mathbf{v}_{h}, q_{h}\right]\right)=-\frac{1}{2 N_{w}}\left\langle\mathbf{v}_{h}, \mathbf{n} \cdot \sigma_{h}\left(\mathbf{u}_{h}^{n+1}, p_{h}^{n+1}\right)\right\rangle_{\Gamma_{L}} \\
-\frac{1}{2 N_{w}}\left\langle\mathbf{u}_{h}^{n+1}, \mathbf{n} \cdot \sigma_{h}\left(\mathbf{v}_{h}, q_{h}\right)\right\rangle_{\Gamma_{L}}+\left(1-\frac{1}{2 N_{w}}\right)\left\langle c \rho \mathbf{v}_{h} \cdot \mathbf{n}, \mathbf{u}_{h}^{n+1} \cdot \mathbf{n}\right\rangle_{\Gamma_{L} \cup \Gamma_{O}} \\
+\frac{1}{2 N_{w}} \beta \frac{\mu_{p}}{l_{p}}\left\langle\mathbf{v}_{h}, \mathbf{u}_{h}^{n+1}\right\rangle_{\Gamma_{L}}+\frac{1}{N_{w}} \beta \frac{\mu_{p}}{l_{p}}\left[\sum_{k=n-N_{w}+2}^{n}\left\langle\mathbf{v}_{h}, \mathbf{u}_{h}^{k}\right\rangle_{\Gamma_{L}}-\frac{1}{2}\left\langle\mathbf{v}_{h}, \mathbf{u}_{h}^{n-N_{w}+1}\right\rangle_{\Gamma_{L}}\right] \\
-\frac{1}{N_{w}}\left[\sum_{k=n-N_{w}+2}\left\langle\mathbf{v}_{h}, \mathbf{n} \cdot \sigma_{h}\left(\mathbf{u}_{h}^{k}, p_{h}^{k}\right)\right\rangle_{\Gamma_{L}}-\frac{1}{2}\left\langle\mathbf{v}_{h}, \mathbf{n} \cdot \sigma_{h}\left(\mathbf{u}_{h}^{n-N_{w}+1}, p_{h}^{n-N_{w}+1}\right)\right\rangle_{\Gamma_{L}}\right] \\
-\frac{1}{N_{w}}\left[\sum_{k=n-N_{w}+2}^{n}\left\langle\mathbf{u}_{h}^{k}, \mathbf{n} \cdot \sigma_{h}\left(\mathbf{v}_{h}, q_{h}\right)\right\rangle_{\Gamma_{L}}-\frac{1}{2}\left\langle\mathbf{u}_{h}^{n-N_{w}+1}, \mathbf{n} \cdot \sigma_{h}\left(\mathbf{v}_{h}, q_{h}\right)\right\rangle_{\Gamma_{L}}\right] \\
\sum_{k=n-N_{w}+2}\left\langle c \rho \mathbf{v}_{h} \cdot \mathbf{n}, \mathbf{u}_{h}^{k} \cdot \mathbf{n}\right\rangle_{\Gamma_{L} \cup \Gamma_{O}}-\frac{1}{2 N_{w}}\left\langle c \rho \mathbf{v}_{h} \cdot \mathbf{n}, \mathbf{u}_{h}^{n-N_{w}+1} \cdot \mathbf{n}\right\rangle_{\Gamma_{L} \cup \Gamma_{O}} . \\
L_{B}\left(\left[\mathbf{v}_{h}, q_{h}\right]\right)=\beta \frac{\mu_{p}}{l_{p}}\left\langle\mathbf{v}_{h}, \mathbf{u}_{L}\right\rangle_{\Gamma_{L}}-\left\langle\mathbf{u}_{L}, \mathbf{n} \cdot \sigma_{h}\left(\mathbf{v}_{h}, q_{h}\right)\right\rangle_{\Gamma_{L}}
\end{array}
$$

When a PML is mandatory $B_{P M L}$ must be included in the formulation. Using 22 the discrete bilinear form for the PML can be easily derived:

$$
B_{P M L}\left(\left[\mathbf{u}_{h}, p_{h}\right],\left[\mathbf{v}_{h}, q_{h}\right]\right)=\alpha^{*}\left(\mathbf{v}_{h}, \mathbf{u}_{h}^{n+1}\right)_{\Omega_{P M L}}+\alpha\left(q_{h}, \rho p_{h}^{n+1}\right)_{\Omega_{P M L}} .
$$

The last step for a robust and consistent formulation consists in developing an appropriate stabilization for $B$. On the one hand, one can profit from all terms coming from the stabilization of the incompressible Navier-Stokes. In the present case, the Algebraic Subgrid Scale (ASGS) method for incompressible flows presented in 38 , has been taken as reference. On the other hand, the two pressure terms must be included in the residual of the continuity equation for consistency and the pressure convective term must be added to the stabilization operator. Since linear P1/P1 elements will be used, the second order viscous terms have been removed from the residual and the stabilization operator of the momentum equation. As it will be shown in the next chapter, this extension of the incompressible flow stabilization also prevents the dissipation of the acoustic 
waves:

$$
\begin{array}{r}
B_{S}\left(\left[\mathbf{u}_{h}^{n+1}, p_{h}^{n+1}\right],\left[\mathbf{v}_{h}, q_{h}\right]\right)=\sum_{K} \tau_{1, K}\left(\rho \mathbf{u}_{h}^{n+1} \cdot \nabla \mathbf{v}_{h}+\nabla q_{h}, \rho \delta_{t} \mathbf{u}_{h}^{n+1}\right. \\
\left.+\rho \mathbf{u}_{h}^{n+1} \cdot \nabla \mathbf{u}_{h}^{n+1}-\mu \nabla^{2} \mathbf{u}_{h}^{n+1}-\frac{1}{3} \mu \nabla\left(\nabla \cdot \mathbf{u}_{h}^{n+1}\right)+\nabla p_{h}^{n+1}\right)_{K} \\
+\sum_{K} \tau_{2, K}\left(\rho \nabla \cdot \mathbf{v}_{h}+\frac{1}{c^{2}} \mathbf{u}_{h}^{n+1} \cdot \nabla q_{h}, \rho \nabla \cdot \mathbf{u}_{h}^{n+1}+\frac{1}{c^{2}} \mathbf{u}_{h}^{n+1} \cdot \nabla p_{h}^{n+1}+\frac{1}{c^{2}} \delta_{t} p_{h}^{n+1}\right)_{K},
\end{array}
$$

where $K$ denotes the element domain, and $\tau_{1, K}$ and $\tau_{2, K}$ are suitable stabilization parameters defined in each element, [39]:

$$
\begin{aligned}
\tau_{1, K} & =\left[c_{1} \frac{\nu}{h^{2}}+c_{2} \frac{\left|\mathbf{u}_{h}^{n+1}\right|}{h}\right]^{-1} \\
\tau_{2, K} & =\frac{h^{2}}{c_{1} \tau_{1, K}}
\end{aligned}
$$

\section{Results}

For a proper validation of the present formulation two different scenarios have been taken as reference. First, a $2 \mathrm{D}$ problem consisting in a low speed $\mathrm{Re}=$ 1000 flow around a cylinder has been calculated with the isentropic compressible equations for comparing the CFD results and the acoustic propagation to those provided by an incompressible solver and the Lighthill analogy. Second, a $\mathrm{M}=$ 0.4 flow around a 3D NACA 0012 airfoil has been calculated in order to evaluate the performance of the formulation against a compressible flow solver and the

Ffowcs Williams \& Hawkings (FWH) acoustic analogy. The main advantage of the isentropic compressible formulation is that it can be treated numerically like the incompressible formulation although the flow regime might not be in the incompressible range anymore. From the point of view of an end user, the only further requirement consists in introducing the three following parameters: the gas universal constant $R=8.31 \mathrm{~J} / \mathrm{Kmol}$, the molar mass, the sound propagation speed of the working gas and the bulk temperature. In both cases the values of air at room temperature have been considered $\left(\mathcal{M}=28.97 \mathrm{~g} / \mathrm{mol}, c_{0}=343\right.$ $\mathrm{m} / \mathrm{s}$ and $\left.T_{0}=293.15 \mathrm{~K}\right)$. 


\subsection{Aerodynamic sound radiated by flow past a cylinder. $M=0.0583$}

The first benchmark case consists in a 2D flow around a $D=0.3$ cylinder which allows evaluating the aeolian tones of a low Mach viscous flow, 40. The incident velocity of 20 leads to a Reynolds and Mach numbers at the far field (away from the cylinder) of $\mathrm{Re}=1000$ and $\mathrm{M}=0.0583$ for a sound speed of $c_{0}=343$ (all units are in SI). The problem has been solved in an unstructured mesh of nearly 1 million triangular linear elements using equal interpolation for velocity and pressure, with a size of $3 \cdot 10^{-3} D$ near the cylinder surface. The case has been run up to $1.5 \mathrm{~s}$ with a time step $\delta t=1 \cdot 10^{-3} \mathrm{~s}$, departing from an initial incompressible solution in order to ease the initial convergence of the iterative solver. In fact, the same solver does not yield convergence when the problem is computed with the incompressibility condition, even when departing from a converged solution. For the weak imposition of boundary conditions it has been enough taking a penalty parameter $\beta=1$.

The original case in [40] was computed with the incompressible Navier-Stokes equations and Lighthill's analogy in the frequency domain (Helmholtz equation). Regarding the CFD, this calculation provides a shedding frequency of $15.3 \mathrm{~Hz}$, whereas the present formulation has obtained a very similar value of $15.6 \mathrm{~Hz}$. The fully developed velocity profiles are compared in Fig. 4. This very good fitting between both velocity profiles does not only assess the accuracy of the isentropic compressible equations at low Mach regimes, but illustrates the possibility of replacing the incompressible monolithic solvers when their convergence is not satisfactory even if the acoustics are not relevant. Moreover, it confirms the good performance of the weakly imposed inlet condition. Of course, one may think that, despite this huge benefit, the compressibility brings the big drawback of waves being reflected by the boundaries and polluting the flow solution. However, Fig. 5 shows that this inconvenience is completely resolved by the previously presented boundary conditions as no reflections are observed on the external boundaries. This plot also validates qualitatively the acoustic propagation at the far field. The present formulation is capable of capturing the anisotropy of the aeolian tones as well as the amplitude of the acoustic waves. 
All three curves show a very good fitting in the near field, but some discrepancies appear beyond the $50 \mathrm{~m}$. The stripped curve shows the importance of the extension of the incompressible stabilization terms presented in Eq. (33). If the compressible terms are not taken into account in the residual, the wave dominant but causes a drastic dissipation of the waves at the far-field. When the full stabilization is deployed, the resulting curve approximates the reference one more accurately. The phase error can be associated to the shedding frequency differential between both formulations, whereas the small amplitude difference gion, where the flow stagnation hypothesis is not fulfilled, and which can lead to an over-prediction of the amplitude of the acoustic signal, [16].

\subsection{Aerodynamic sound radiated by flow past an airfoil. $M=0.4$}

The second benchmark case consists in a 3D flow around a NACA 0012 airfoil with an angle of attack of $5^{\circ}$, 41]. The flow Reynolds number based on the airfoil chord $(d=0.1524)$ is $\operatorname{Re}_{c}=408000$ whereas the incident Mach number is $\mathrm{M}=0.4$. The problem has been solved in an unstructured mesh of nearly 20 million tetrahedral linear elements using equal interpolation for velocity and pressure, with a size of $4 \cdot 10^{-4}$ on the leading edge and $6.5 \cdot 10^{-4}$ on the rest of the airfoil surface (all units are in SI). The case has been run up to $0.050 \mathrm{~s}$ with a time step $\delta t=10^{-5} \mathrm{~s}$, departing from an initial incompressible solution in order to ease the initial convergence of the iterative solver. For the weak imposition of boundary conditions a penalty parameter $\beta=125$ has been taken. Unlike the previous low-speed flow, the present case generates an airjet that cannot be dissipated before reaching the outlet, for which a PML has been placed in this region. On the external boundaries the flow field has been prescribed separately following the presented method.

The original case in [4] was computed with a compressible Large Eddy Simulation (LES) for the flow scales and the Ffowcs Williams \& Hawkings (FWH) 


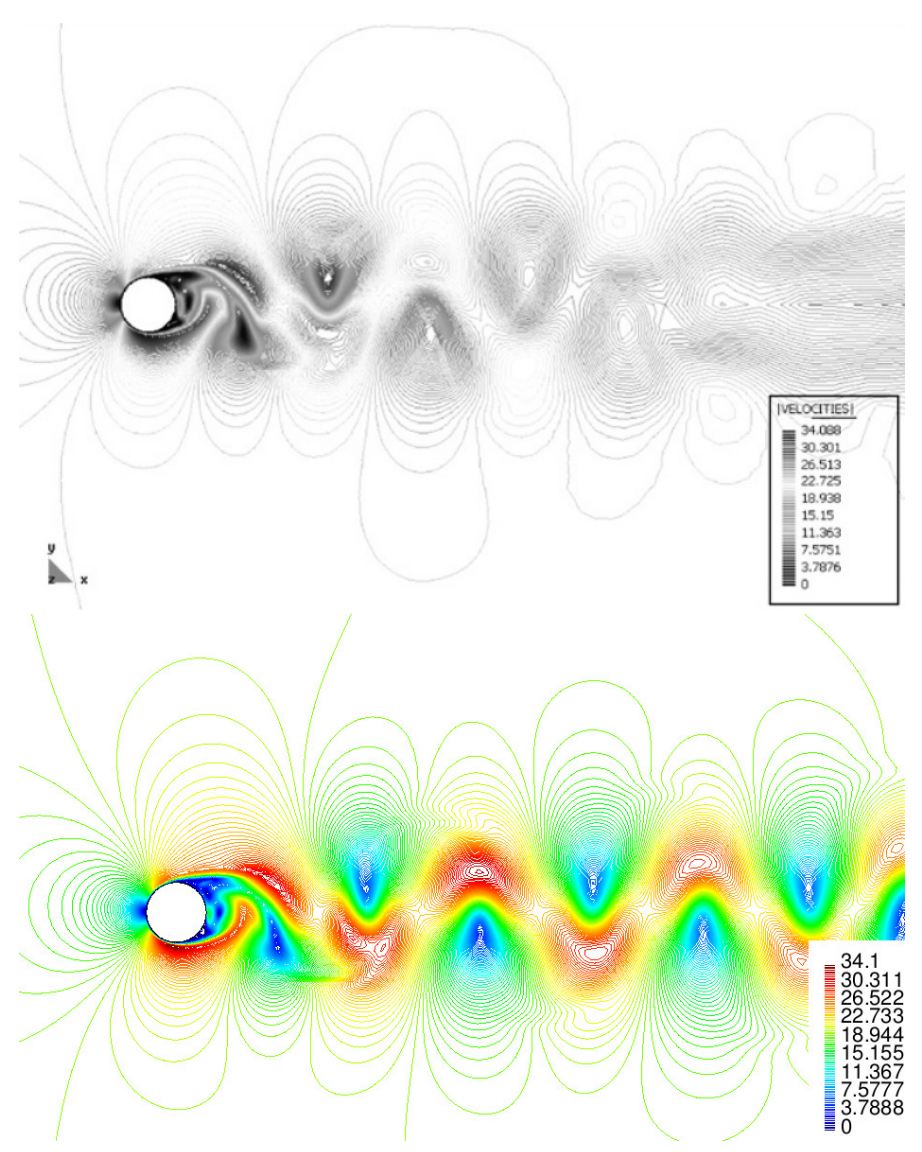

Figure 4: Incompressible flow velocity (top), isentropic compressible velocity (bottom). ference mesh has been able to capture high frequencies up to more than the Helmholtz number $k d=40$, which is approximately $15 \mathrm{kHz}$. This spatial resolution, together with a much smaller time step, could not be reproduced with an unstructured mesh of tetrahedral elements under a reasonable cost taking 350 into account the available resources. Since the object of the present work does not consist in assessing the performance of the solver in specific mesh typologies or in reproducing all the details of a particular problem, but in establishing a general framework for the calculation of a wide range of flows, the goal of this analysis has been restricted to the following points: the suitability of the present 


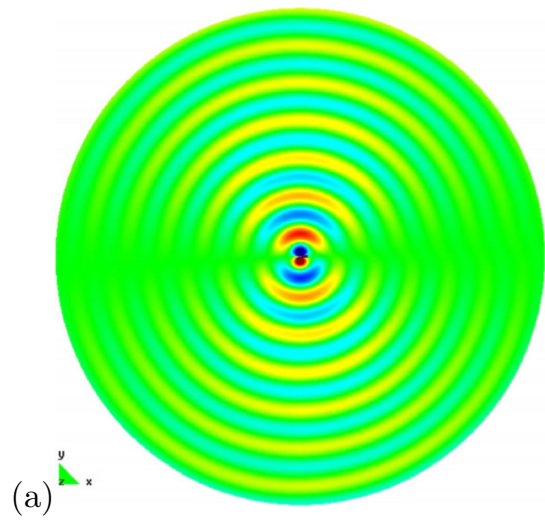

(b)

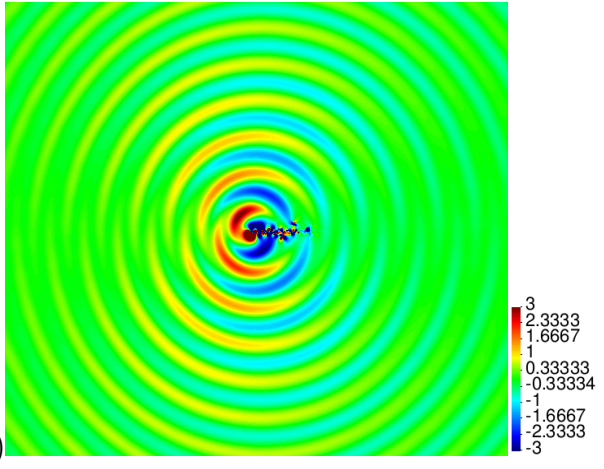

Figure 5: Imaginary component of the acoustic pressure (a), isentropic compressible pressure (b).

isentropic compressible formulation for reproducing the mean flow patterns at $\mathrm{M}=0.4$, the proper propagation of the captured acoustic modes, and finally the validation of the proposed boundary conditions.

The validation of the formulation in regard to the mean flow variables is perhaps the most demanding aspect of this simulation. The lack of resolution for properly capturing the boundary layer around the airfoil makes it difficult to evaluate the aerodynamics of the present formulation. In a first approach, the surface of the airfoil was prescribed a non-slip boundary condition, but it yielded an early separation of the boundary layer. In order to prevent this scenario, a wall-law with both buffer and logarithmic regions has been prescribed and the result in Fig. 7 has been obtained:

Although the mean velocity field values are properly reproduced, the boundary layer still suffers an early detachment from the airfoil. In order to analyse in what extent the mesh element size, and not the formulation, was the reason for this discrepancy, the same problem has been run in a $2 \mathrm{D}$ section of the original domain using a much finer mesh. Fig. 7p shows that the element size around the wall was indeed the cause of the early boundary layer detachment.

The same dependence on the mesh resolution can be found in the capturing of the acoustic modes. However, in this case the lack of accuracy can be restricted 


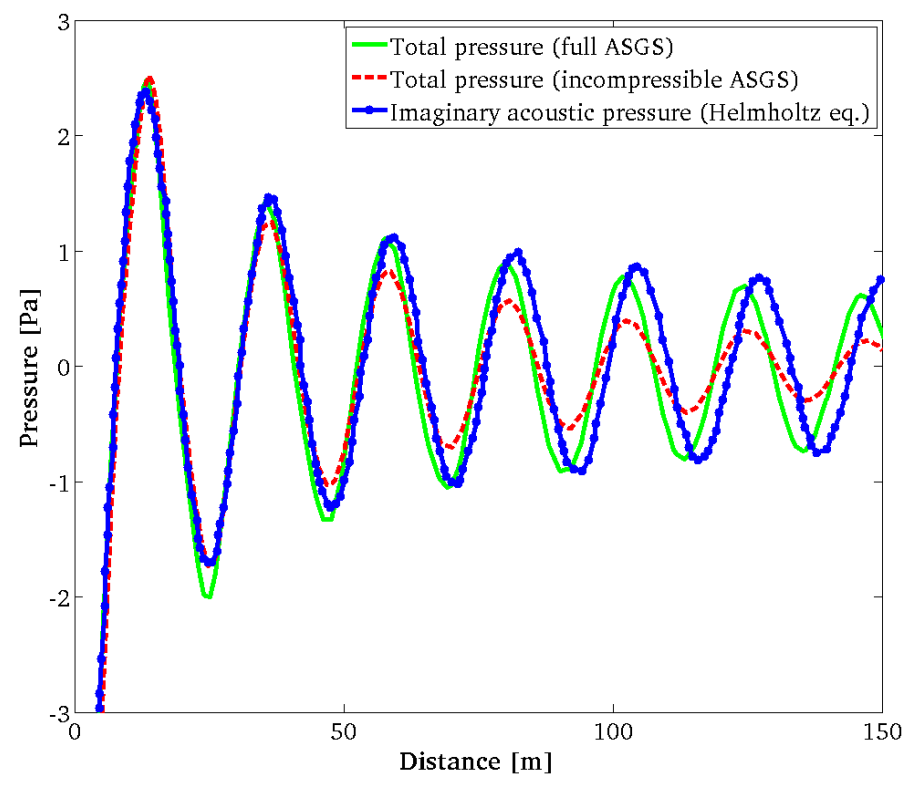

Figure 6: Wave propagation at 90 with respect to the $\mathrm{x}$-axis.

to a truncation of the frequency spectrum. For this reason, the calculated wave

375 propagation in Fig. 8a can only validated against two profiles corresponding to the acoustic modes $k d=2.45$ and $k d=4.91$ of [41, which correspond to frequencies that can be captured by the present mesh. Fig. 8 also shows how the wave propagation in the nearest region around the airfoil is not visible due to the presence of the much larger aerodynamic scale, but beyond a certain point it arises with the same pattern of the solution calculated with the FWH acoustic analogy.

The behavior of waves abandoning the domain is one of the main concerns in compressible flow calculations. In the previous case, due to the low Mach and Reynolds regime, the size of the near-field region under influence of the flow perturbations was very small compared to the domain size, for which the separated prescription of boundary conditions considering a mean flow component and an acoustic variation was conceptually very clear. However, in the present case real far-field stagnation conditions cannot be reached within a reasonably big domain and the aerodynamics pressure field is not yet uniform on the ex- 


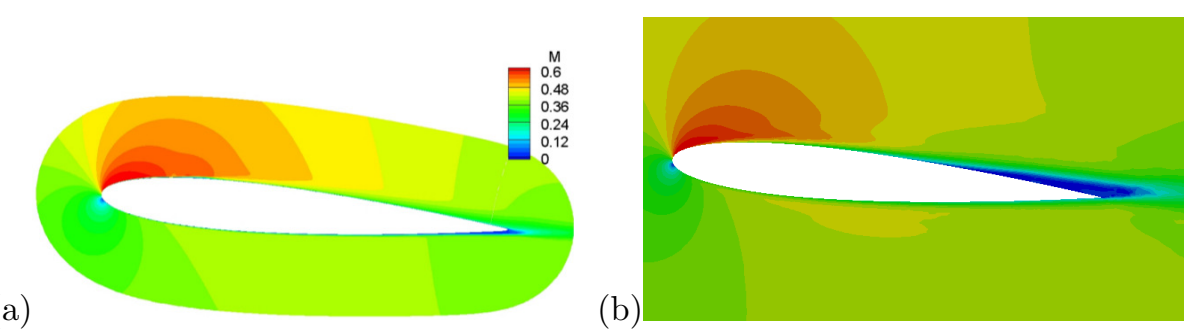

(a)

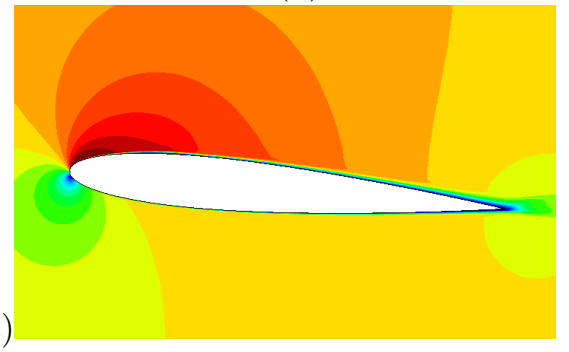

Figure 7: Contours of time-averaged flow velocity. Reference (a), calculated in a 3D domain (b), calculated in a $2 \mathrm{D}$ domain (b).

ternal boundaries, see Fig. 2. In spite of this theoretical drawback, the method manages to separate the two scales perfectly by updating the mean value at each time step, which allows the waves to cross $\Gamma_{L}$ without any spurious reflection, see Fig. 8b. On the other hand, the implemented PML on the outlet absorbs perfectly the incoming waves as well as the noise produced by the airjet. It can be also observed how the flow pressure field is not exactly flat on $\Gamma_{L}$ but no spurious reflections appear. This is possible because the present method is able to account for variations in the mean flow variables, but they must be smooth enough so they do not interfere with the acoustic field, otherwise spurious reflections may appear. Therefore, the truncation of the domain is not so immediate in this case since it must be assessed in advance that the far-field variations are acceptable, keeping always the computational cost in mind. Someone may argue that this compromise could be avoided by using a PML on all external boundaries. Unfortunately this is only possible in solvers that compute the acoustic and the flow scales separately. In compressible formulations where the full variables are solved in a single calculation, like the present one, the inlet cannot be in contact with a PML. Moreover, the use of a global PML on all 
other external boundaries can lead to an unaffordable computational cost in big 3D cases with large wavelengths, for which the use of a non-radiating boundary condition has been prioritized.

\section{Conclusions}

The presented FEM formulation offers a simplified framework for dealing with subsonic adiabatic gas flows without facing the numerical inconveniences and the high computational cost of the state-of-the-art compressible flow formulations. The minimal implementation cost when departing from a monolithic incompressible solver makes this approach very attractive for solving aeroacoustic problems where heat transfer can be neglected. Moreover, its validation against the incompressible Navier-Stokes equations for a low-Mach regime has shown its suitability for boosting the solver in problems where acoustics are not relevant.

From a physical point of view, the developed numerical method has successfully reproduced both the acoustics of the incompressible Lighthill analogy and the FWH analogy with compressible flow, ranging from $\mathrm{M}=0.058$ to $\mathrm{M}=0.4$. Therefore, one of the main goals of this research, the development of a general numerical framework for all isentropic gas flows has been successfully accom-

${ }_{225}$ plished. In this sense, the presentation of a novel method for prescribing separate boundary conditions for the aerodynamic and the acoustic components strives in the same direction of offering a general solution to the problem of spurious wave reflection in aeroacoustic calculations. On the most problematic boundary for such cases, the inlet, this formulation manages to prescribe an incoming velocity while being transparent to the exiting acoustic waves. Moreover, it is compatible with any kind of non-radiating boundary condition and can be combined with a PML on the outlet in case of highly convective jets. 


\section{Acknowledgements}

The authors gratefully acknowledge the computer resources, technical exper-

435 BSC) and the financial support to CIMNE via the CERCA Programme/Generalitat de Catalunya. This work has been partially supported by the EU-FET grant EUNISON 308874. Moreover, the first author would also like to thank the Agència de Gestió d'Ajuts Universitaris i de Recerca for the predoctoral FI

[5] C. A. Bayona Roa, J. Baiges, R. Codina, Variational multi-scale finite element approximation of the compressible navier-stokes equations, International Journal of Numerical Methods for Heat \& Fluid Flow 26 (3/4) (2016) 1240-1271. 
[6] C. Bailly, C. Bogey, O. Marsden, Progress in direct noise computation, noise notes 9 (3) (2010) 31-48.

[7] M. J. Lighthill, On sound generated aerodynamically. i. general theory, Proceedings of the Royal Society of London. Series A. Mathematical and Physical Sciences 211 (1107) (1952) 564-587.

[8] N. Curle, The influence of solid boundaries upon aerodynamic sound, Proceedings of the Royal Society of London. Series A. Mathematical and Physical Sciences 231 (1187) (1955) 505-514.

[9] J. F. Williams, D. L. Hawkings, Sound generation by turbulence and surfaces in arbitrary motion, Philosophical Transactions of the Royal Society of London. Series A, Mathematical and Physical Sciences 264 (1151) (1969) 321-342.

[10] J. Hardin, D. Pope, An acoustic/viscous splitting technique for computational aeroacoustics, Theoretical and Computational Fluid Dynamics 6 (56) (1994) 323-340.

[11] W. Z. Shen, J. N. Sørensen, Aeroacoustic modelling of low-speed flows, Theoretical and Computational Fluid Dynamics 13 (4) (1999) 271-289.

[12] C. Bogey, C. Bailly, D. Juvé, Computation of flow noise using source terms in linearized euler's equations, AIAA journal 40 (2) (2002) 235-243.

[13] C. Bailly, D. Juve, Numerical solution of acoustic propagation problems using linearized euler equations, AIAA journal 38 (1) (2000) 22-29.

[14] J. H. Seo, Y. J. Moon, Linearized perturbed compressible equations for low mach number aeroacoustics, Journal of Computational Physics 218 (2) (2006) 702-719.

[15] R. Ewert, W. Schröder, Acoustic perturbation equations based on flow de485 composition via source filtering, Journal of Computational Physics 188 (2) (2003) 365-398. 
[16] M. Kaltenbacher, S. Zörner, A. Hüppe, P. Sidlof, 3d numerical simulations of human phonation, in: Proceedings of the 11th world congress on computational mechanics-WCCM XI, 2014.

[17] O. Guasch, P. Sánchez-Martín, A. Pont, J. Baiges, R. Codina, Residualbased stabilization of the finite element approximation to the acoustic perturbation equations for low mach number aeroacoustics, International Journal for Numerical Methods in Fluids.

[18] M. Roger, The acoustic analogy some theoretical background, Lecture series-van Kareman Institute for fluid dynamics 2 (2000) A1-A32.

[19] O. Guasch, A. Pont, J. Baiges, R. Codina, Concurrent finite element simulation of quadrupolar and dipolar flow noise in low mach number aeroacoustics, Computers \& Fluids 133 (2016) 129-139.

[20] R. Codina, J. Baiges, Weak imposition of essential boundary conditions in the finite element approximation of elliptic problems with nonmatching meshes, International Journal for Numerical Methods in Engineering 104 (7) (2015) 624-654.

[21] H. Espinoza, R. Codina, S. Badia, A sommerfeld non-reflecting boundary condition for the wave equation in mixed form, Computer Methods in Applied Mechanics and Engineering 276 (2014) 122-148.

[22] P. H. Oosthuizen, W. E. Carscallen, Introduction to compressible fluid flow, CRC Press, 2013.

[23] M. Avila, J. Principe, R. Codina, A finite element dynamical nonlinear subscale approximation for the low mach number flow equations, Journal of Computational Physics 230 (22) (2011) 7988-8009.

[24] P. Fosso, H. Deniau, N. Lamarque, T. Poinsot, et al., Comparison of outflow boundary conditions for subsonic aeroacoustic simulations, International journal for numerical methods in fluids 68 (10) (2012) 1207-1233. 
[25] V. Granet, O. Vermorel, T. Léonard, L. Gicquel, T. Poinsot, Comparison of nonreflecting outlet boundary conditions for compressible solvers on unstructured grids, AIAA journal 48 (10) (2010) 2348-2364.

[26] K. W. Thompson, Time dependent boundary conditions for hyperbolic systems, Journal of computational physics 68 (1) (1987) 1-24.

[27] T. J. Poinsot, S. Lele, Boundary conditions for direct simulations of compressible viscous flows, Journal of computational physics 101 (1) (1992) 104-129.

[28] R. Prosser, Improved boundary conditions for the direct numerical simulation of turbulent subsonic flows. i. inviscid flows, Journal of Computational Physics 207 (2) (2005) 736-768.

[29] R. Prosser, Towards improved boundary conditions for the dns and les of turbulent subsonic flows, Journal of Computational Physics 222 (2) (2007) $469-474$.

[30] C. S. Yoo, H. G. Im, Characteristic boundary conditions for simulations of compressible reacting flows with multi-dimensional, viscous and reaction effects, Combustion Theory and Modelling 11 (2) (2007) 259-286.

[31] J.-P. Berenger, A perfectly matched layer for the absorption of electromagnetic waves, Journal of computational physics 114 (2) (1994) 185-200.

[32] F. Q. Hu, A perfectly matched layer absorbing boundary condition for linearized euler equations with a non-uniform mean flow, Journal of Computational Physics 208 (2) (2005) 469-492.

[33] C. K. Tam, Z. Dong, Radiation and outflow boundary conditions for direct computation of acoustic and flow disturbances in a nonuniform mean flow, Journal of Computational Acoustics 4 (02) (1996) 175-201.

[34] C. Bogey, C. Bailly, Three-dimensional non-reflective boundary conditions for acoustic simulations: far field formulation and validation test cases, Acta Acustica united with Acustica 88 (4) (2002) 463-471. 
[35] M. Juntunen, R. Stenberg, Nitsches method for general boundary conditions, Mathematics of computation 78 (267) (2009) 1353-1374.

[36] R. Codina, J. Baiges, Approximate imposition of boundary conditions in immersed boundary methods, International Journal for Numerical Methods in Engineering 80 (11) (2009) 1379-1405.

[37] H. Takemoto, P. Mokhtari, T. Kitamura, Acoustic analysis of the vocal tract during vowel production by finite-difference time-domain method, The Journal of the Acoustical Society of America 128 (6) (2010) 37243738 .

[38] R. Codina, On stabilized finite element methods for linear systems of convection-diffusion-reaction equations, Computer Methods in Applied Mechanics and Engineering 188 (1) (2000) 61-82.

[39] R. Codina, J. Principe, O. Guasch, S. Badia, Time dependent subscales in the stabilized finite element approximation of incompressible flow problems, Computer Methods in Applied Mechanics and Engineering 196 (21) (2007) 2413-2430.

[40] O. Guasch, R. Codina, An algebraic subgrid scale finite element method for the convected helmholtz equation in two dimensions with applications in aeroacoustics, Computer Methods in Applied Mechanics and Engineering 196 (45) (2007) 4672-4689.

[41] W. R. Wolf, S. K. Lele, Trailing edge noise predictions using compressible les and acoustic analogy, in: Proceedings of the 17th AIAA/CEAS Aeroacoustics Conference, AIAA Paper, Vol. 2784, 2011, pp. 1-25. 


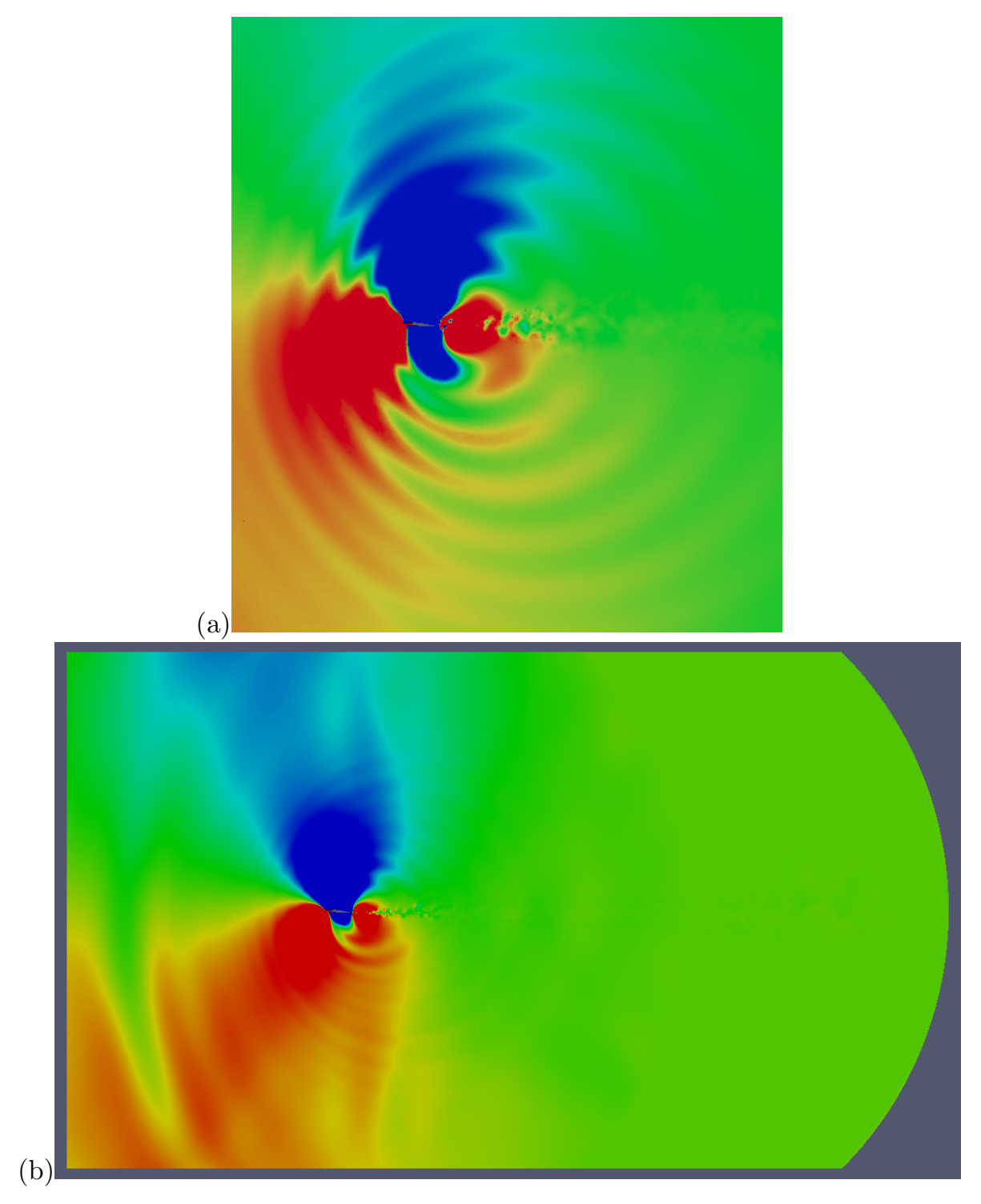

Figure 8: Reference wave propagation contour for $k d=2.45$ (a), reference wave propagation contour for $k d=4.91$ (b), calculated total pressure (c) 